\title{
UNE TOUCHE DE NOIR, UNE TOUCHE DE BLANC. VOLCY FOCARD EXPLIQUE LA CRÉOLISATION À LA RÉUNION
}

\author{
Philipp Krämer
}

Freie Universität Berlin

\begin{abstract}
Résumé
Si la recherche sur les langues créoles au $\mathrm{XIX}^{\mathrm{e}}$ siècle s'inscrit clairement dans le discours racialiste de la philologie contemporaine, le Réunionnais Volcy Focard prend une position exceptionnelle : au lieu des présumées différences raciales, son explication de la créolisation et des structures grammaticales $\mathrm{du}$ créole réunionnais sont d'ordre sociohistorique plutôt que biologiste. Son article "Les origines du patois de l'île Bourbon » ainsi que ses correspondances avec Hugo Schuchardt révèlent une conception du langage humain qui est à la fois très classique et très progressiste en mettant l'accent sur le lexique et l'écrit, plutôt que sur la grammaire parlée, tout en considérant la variation sociale comme champ de recherche principal.
\end{abstract}

\section{Mots-clés}

Langues créoles, La Réunion, racialisme, philologie coloniale, $\mathrm{XIX}^{\mathrm{e}}$ siècle

\section{Abstract}

Even though early works about Creole languages in the late 19th century frequently follow the patterns of the predominant racist discourse in contemporary philology, the texts by Volcy Focard from La Réunion are remarkable exceptions: his analysis of the emergence and structure of the local Creole language are based upon sociohistorical rather than biological explanations, avoiding the widespread idea of alleged racial differences. His article "Les origines du patois de l'île Bourbon" and his correspondence with Hugo Schuchardt display a notion of language which is both very classical and very progressive. Focard focuses on the lexicon and written language less than on spoken grammar while at the same time putting forward sociolectal variation as the most interesting field for linguistic research.

\section{Keywords}

Creole languages, La Réunion, racialism, colonial philology, 19th century

« Corruption », « perturbation grammaticale », « baragouin »: c'est ainsi que le Réunionnais Volcy Focard caractérise la langue créole de l'île dans un texte paru en 1885. Ces attributs peuvent paraître peu surprenants dans une période où toutes les langues du monde doivent, aux yeux de la philologie contemporaine, se mesurer aux normes européennes. On pourrait alors se contenter de ranger ces propos de Focard dans la même catégorie que ceux des linguistes et philologues du XIX ${ }^{\mathrm{e}}$ siècle qui décrivent les langues créoles en véhiculant en même temps l'idéologie de la 
primauté du Vieux Continent sur les cultures dites primitives et arriérées. Mais, comme le disent les narrateurs des contes traditionnels créoles : Zistwar lé mentèr - l'histoire est trompeuse. En effet, le texte de Focard mérite d'être regardé de plus près pour discerner les nuances qui le distinguent des autres travaux de la créolistique du XIX ${ }^{\mathrm{e}}$ siècle. Le travail de Focard donne lieu à des questions souvent négligées par la créolistique actuelle qui met fréquemment l'accent sur l'analyse des textes historiques en tant que sources de "données grammaticales », sur la discussion de la fiabilité de ces données et sur une comparaison des résultats des auteurs historiques avec les connaissances actuelles des structures linguistiques. Si ces questions sont évidemment loin d'être marginales, il convient d'y ajouter aussi la dimension épistémologique.

\section{KOSA BANN KRÉOLIST I MAZINÉ ?}

Il est d'ores et déjà établi que l'étude des langues créoles au XIX ${ }^{\mathrm{e}}$ siècle suit largement le modèle racialiste ${ }^{2}$ tel qu'il se manifeste dans la plupart des autres domaines philologiques de l'époque ${ }^{3}$. Vers la fin du XIX ${ }^{e}$ siècle, les travaux sur les langues créoles se multiplient non seulement avec l'œuvre monumentale d'Hugo Schuchardt en Autriche, mais aussi en France et dans la sphère coloniale francophone ainsi qu'au Portugal. En gros, l'existence des créoles était expliquée par une prétendue infériorité raciale du « Noir » qui serait incapable d'adopter la langue élaborée et complexe que serait le français. La simplicité intellectuelle serait responsable des simplifications syntaxiques et morphologiques, la différence physique se serait traduite par des changements phonologiques, et la paresse notoire mènerait à une réduction des formes grammaticales à tout niveau.

René de Poyen-Bellisle, par exemple, écrit sa thèse de doctorat sur le créole antillais en mettant l'accent sur les rapports entre phonologie et physionomie :

Ici l'élément physiologique entre en jeu. L'esclave n'essaie pas pour un seul instant d'assimiler les nouveaux sons qu'il entend à ceux qu'il connaît déjà [...]. Ce qu'il a à faire c'est d'essayer de parler comme son maître. Et il essaie. Mais il n'appartient pas à la même race, son appareil vocal n'est pas le même, ses lèvres sont différentes. De là les sons articulés par le Blanc subiront certaines transformations quand ils sont répétés par le nègre. (Poyen-Bellisle 1894, p. 14-15).

1 «Que pensent les créolistes?»

$2 C f$. Todorov (1989) pour une différenciation judicieuse entre racialisme comme modèle discursif qui opère avec la conviction d'une hiérarchie entre les supposées races humaines, et racisme comme pratique discriminatoire, voire violente. S'il n'est pas toujours facile de faire la distinction, étant donné que la production scientifique est elle-même une forme de pratique, une telle différenciation peut aider à discerner réflexion sous-jacente et acte.

$3 C f$. Messling (2012) pour une vue d'ensemble des implications de la philologie au XIX siècle dans les discours racialistes. $C f$. Krämer (2014a) pour une analyse détaillée des fondements épistémologiques de la créolistique française vers la fin du XIX ${ }^{\mathrm{e}}$ siècle. 
Poyen-Bellisle présume des qualités physiologiques qui seraient différentes d'une race à l'autre et qui servent d'explication pour les altérations phonologiques dans le processus de créolisation. Selon Charles Baissac, auteur d'une Étude sur le patois créole mauricien, les " Noirs » auraient notamment des déficiences intellectuelles et morales qui seraient à l'origine des structures et du lexique créole :

[...] brusquement placés par l'esclavage en présence du monde d'idées nouvelles pour eux que portait en elle la langue française, nos noirs se bouchèrent résolument les yeux et les oreilles, et, en dehors du cercle étroit de la vie matérielle, ils voulurent tout ignorer, se sentant incapables de rien comprendre. (Baissac 1880, p. VIII)

Nous rencontrons dans ce passage un nombre de caractéristiques typiquement attribuées aux « Noirs », comme un prétendu manque d'intelligence et une tendance à l'obstination, et ces caractéristiques seraient à l'origine de la créolisation. Baissac en tire la conclusion que le créole serait inapte surtout aux réflexions abstraites, philosophiques, historiques et littéraires.

Julien Vinson, linguiste français qui atteint une notoriété dans sa discipline, synthétise les idées reçues des créoles en les définissant comme « adaptation[s] d'une langue, et surtout, en fait, d'une langue indo-européenne, au génie pour ainsi dire phonétique et grammatical d'une race linguistiquement inférieure » (1884, p. 345).

C'est ainsi que les auteurs cultivent l'image de la simplicité et de l'infériorité des créoles - image qui s'est enracinée dans la réflexion sur les créoles à tel point qu'elle ressurgit régulièrement dans les débats actuels tant scientifiques que médiatiques.

\section{VOLCY FOCARD, KISA I LÉ ? ${ }^{4}$}

Si certains de nos contemporains ont du mal à accepter la normalité des créoles, un petit nombre de créolistes les devancent déjà au XIX ${ }^{\mathrm{e}}$ siècle. Parmi eux, l'éminent Hugo Schuchardt, mais aussi le Réunionnais Volcy Focard. Celui-ci a été jusqu'à présent un personnage peu connu dans l'histoire de la philologie. Non seulement il est rarement lu, mais il reste littéralement inconnu du fait que nous avons très peu d'informations sur sa biographie ${ }^{5}$. Nous savons cependant qu'il a été fonctionnaire dans le système juridique réunionnais, d'abord comme secrétaire du Procureur

5 Nous restons même dans le doute à propos de la graphie du prénom de Focard : les deux possibilités « Volsy » et « Volcy » alternent dans la littérature, la seconde version paraissant plus répandue. Malheureusement, la lecture de la correspondance entre Focard et Schuchardt, documents plutôt intimes, ne permet pas non plus de clarification définitive (Krämer 2012, p. 137-138). 
Général, et plus tard comme greffier de la Cour d'Appel ${ }^{6}$. C'est ainsi qu'il écrit à Hugo Schuchardt en 1885 qu'il est en train d'accomplir « un travail ennuyeux - le recueil des Lois en vigueur à La Réunion ${ }^{7}$. Outre l'occupation professionnelle, nous connaissons aussi l'une des grandes passions privées de Focard : il est membre de la Société des Sciences et Arts de la Réunion, dans laquelle il fait partie de la commission des Lettres ${ }^{8}$. Dans cette même société savante, Focard donne un discours sur le créole réunionnais qui sera ensuite publié dans le Bulletin annuel (1885). En effet, La Réunion est parmi les endroits qui produisent le plus de travaux dans cette période fructueuse pour la créolistique que sont les dernières décennies du XIX ${ }^{e}$ siècle. Outre Focard, nous comptons un article d'Auguste Vinson ${ }^{9}$ issu de ses correspondances avec Hugo Schuchardt et publié dans le même Bulletin (1883), puis une étude d'Anatole-Joseph Verrier (1906). Schuchardt lui-même, en dépit de sa prédilection pour les créoles à base portugaise (Stein 2005), les précède avec une petite note dans Romania ${ }^{10}$, et son élève Adolphe Dietrich contribue avec une étude comparative des créoles réunionnais et mauricien (1891).

Parmi ces travaux, celui d'Auguste Vinson (1885), médecin et adepte de Haeckel, se démarque par sa dimension naturaliste en se rattachant au biologisme prépondérant du $\mathrm{XIX}^{\mathrm{e}}$ siècle qui se cristallise dans le conglomérat généalogico-historique du racialisme. Si Auguste Vinson assume le ralliement de la créolistique réunionnaise au discours prédominant, Focard nous présente une explication toute différente de la créolisation. Ceci est d'autant plus remarquable qu'il connaît personnellement Auguste Vinson - tous deux sont membres de la société savante - et que le texte de Focard parait deux ans seulement après celui de Vinson. Très peu d'autres travaux de Focard sont aujourd'hui accessibles. Il est difficile de dire combien Focard était actif dans la Société des Sciences et Arts et combien de fois il a effectivement présenté des travaux. On peut noter cependant que le Bulletin de la société contient un texte de Focard dès l'édition de l'année 1862 et qu'il a donc été membre pendant une longue période. Dans ce texte, Focard analyse les événements consécutifs à l'abolition de l'esclavage à La Réunion et il se présente ainsi comme un personnage aux intérêts variés qui ne portent pas seulement sur le droit et la philologie mais aussi sur l'histoire locale.

6 Voir la liste des membres dans le Bulletin de la Société des sciences et arts de l'île de la Réunion (année 1862, p. 271 ; année 1883, p. 6).

7 Voir la lettre de Volcy Focard à Hugo Schuchardt, datée du 14 avril 1885 ; No. 03085 dans les archives de l'Université de Graz (Hugo-Schuchardt-Archiv). Voir aussi Wolf (1993, p. 172), Krämer (2012, p. 140-141).

8 Voir la composition des commissions indiquée dans le Bulletin de la Société des sciences et arts de l'île de la Réunion (année 1862, p. 269).

9 À ne pas confondre avec Julien Vinson, mentionné plus haut.

10 Schuchardt (1882). Ce texte est reproduit aussi dans le Bulletin de la Société des sciences et arts de l'île de la Réunion en 1884. 


\section{KOMAN FOCARD I ARKONT ANOU ZISTWAR KRÉOL LARÉNYON ? ${ }^{11}$}

En effet, Focard écrit une histoire de la créolisation qui est inhabituelle pour l'époque. Certes, il dit lui aussi que le vrai créole serait parlé par les « Noirs »:

Mais ce n'est pas dans ce langage prétentieux, dans ce boursoufflage sans règles des petits créoles, ni dans le baragouin des cafres, qu'il faut chercher le véritable patois de Bourbon. Il est chez les noirs indigènes, surtout chez les négresses. C'est là qu'il se trouve dans toute sa verdeur et sa vivacité. (Focard 1885, p.189)

Il faut cependant se garder d'y voir une répétition du motif répandu de la création du créole par les « Noirs » et par leur condition raciale. Selon Focard, il y a tout à fait un créole «typiquement noir », tout comme il y a un créole « cafre » et un créole «blanc». Effectivement, Focard place les premiers colons européens au début de la créolisation. Celle-ci serait une conséquence non pas de l'infériorité noire, mais d'un manque de scolarisation - donc des circonstances sociopolitiques qui ne concernent, au début de la colonisation, que les descendants des Européens. Quand Hugo Schuchardt demande à Focard de lui expliquer l'histoire linguistique de la Réunion, Focard lui répond dans une lettre : « À cette époque, on ne montrait pas à lire dans les Colonies $»^{12}$. Ce sont d'abord les enfants des colons - donc des enfants blancs - qui ont altéré la langue parce qu'ils ne connaissaient pas suffisamment la norme écrite. Ce n'est que dans un deuxième temps que les enfants noirs adopteront cette langue déjà transformée et qu'ils contribueront à son développement. Focard écrit :

Plus tard les enfants des noirs sont venus se mêler aux enfants des blancs, de ces marins et employés, Les petits créoles d'aujourd'hui - et de concert, ils ont créé cette sorte d'idiome qui est notre patois indigène ${ }^{13}$.

Ces deux pas de la créolisation anticipent sur la conception de la «basilectalisation » qui est devenue, dans la seconde moitié du XXe siècle, une théorie établie pour l'émergence du créole réunionnais. Celui-ci se serait formé à partir du français informel et dialectal des premiers colons et des structures de plus en plus créolisées se seraient formées au fur et à mesure que l'esclavage de masse entravait l'acquisition du français par les esclaves (Chaudenson 1981, p. 153 ; Mufwene 2005, p. 32). Si Focard parle du « vrai » créole, il y voit donc la variété la plus basilectale sans que l'existence de celle-ci s'explique nécessairement par des différences intellectuelles et physiques de ses locuteurs.

Il faut surtout noter l'expression " de concert », car c'est ce petit passage qui exclut l'application directe de l'idéologie racialiste : si les enfants blancs et noirs ont contribué à la transformation de la langue, une prétendue différence

11 «Comment Focard nous explique-t-il l'histoire du créole réunionnais?»

12 Lettre de Focard à Schuchardt datée du 12 mai 1885, No. 3086.

13 Ibid. ; voir aussi Krämer (2012, p. 141-142). 
raciale ne peut pas avoir joué de rôle prépondérant. Focard connaît tout à fait les caractéristiques qu'on attribue couramment aux « Noirs », cette représentation abstraite composée d'un nombre de stéréotypes qui réapparaissent aussi dans le texte. Il y ajoute cependant aussi de nouvelles qualités positives, par exemple en postulant que la concision du créole serait due à la "rapidité de la pensée " du « Noir » (1885, p. 190). Si beaucoup d'autres créolistes reconnaissent une tendance du créole à la concision, ils l'expliquent habituellement par la prétendue tendance des « Noirs » à la fainéantise. Qui plus est, Focard ne tire pas de conclusion directe de ces caractéristiques prototypiques, surtout pas dans son idée de la créolisation, et il évite la séparation binaire absolue constituante pour la pensée racialiste. Tout comme Hugo Schuchardt, Focard s'intéresse beaucoup à la variation interne de la langue (Vintilă-Rădulescu 1976, p. 130). Il compare les variétés créoles des différents groupes sociaux de l'île, dont les personnes d'origine malgache ou mozambicaine, mais aussi les Petits Créoles ou Petits Blancs $^{14}$. Ce groupe social, propre à la démographie réunionnaise, est d'origine européenne, mais n'a pas profité du système colonial. Issus d'une communauté de travailleurs défavorisés depuis le début du peuplement, ces Petits Blancs ne sont jamais entrés dans le système d'exploitation esclavagiste et ne sont pas arrivés à la prospérité au même titre que les grands propriétaires (Bourquin 2005, p. 16 $s q q$.). Avant le développement des infrastructures modernes favorisant la mobilité spatiale et sociale, ils vivaient surtout à l'intérieur de l'île, sur les hauteurs, dans un certain isolement. Ceci a mené, dans la société réunionnaise, à un éloignement du terme blanc de sa signification exclusivement ethnique au point de désigner une catégorie sociale plutôt que raciale (Chaudenson 1974, p. 87, 93-94). Focard (1885, p. 182) observe donc que le créole « [...] n'est point parlé absolument de la même manière par les diverses castes ou classes de la population ", les Petits Blancs inclus. Les termes « castes » et « classes » sont bien significatifs ici, étant donné que l'idée de la stratification sociale commence à se répandre à la fin du $\mathrm{XIX}^{\mathrm{e}}$ siècle et que ces concepts seront aussi appliqués à la variation linguistique.

C'est donc surtout au niveau social qu'il faut chercher l'explication focardienne des structures créoles. En phonologie, par exemple, il fait une différence subtile : « Nous avons dit plus haut, que les petits créoles abusaient de l'accent grave ; les noirs indigènes, eux, abusent de l'accent aigu. » (1885, p. 190). Focard déduit la façon de parler d'un mode de comportement - d'un abus -, ce qui fait d'abord penser aux arguments racialistes qui font le lien entre un présupposé caractère prototypique de la race et la grammaire. Pourtant, ce comportement est maintenant attribué aux deux groupes de la même façon, cette tendance à l'abus ne peut donc pas être due aux conditions raciales. Elle dépend effectivement des conditions sociales tout court : selon Focard, les Petits Blancs cherchent à se démarquer

14 Comme la grande majorité des créolistes, cependant, Focard « laisse toujours de côté les indiens. » (Focard 1885, p. 182). 
socialement en conservant un certain style linguistique qui est censé les rapprocher du reste de la communauté «Blanche ». Si celle-ci est séparée linguistiquement entre privilégiés francophones et défavorisés créolophones, le seul fait d'être " blanc » ne peut pas être à la base des structures linguistiques du sociolecte des Petits Blancs. Il est tout à fait remarquable, surtout en regard des positions des autres créolistes, que Focard n'attribue pas un «meilleur » créole aux Petits Blancs. Il ne transfère pas l'idée de la supériorité blanche dans sa pratique de créoliste. Si le modèle de Focard n'exclut pas du tout les hiérarchies sociales, il ne se fonde clairement pas sur une hiérarchie raciale.

Schuchardt, lui, procède d'une manière similaire en relevant la variété des « Noirs du Mozambique », donc d'un groupe qui est communément inclus dans la seule catégorie des "Noirs ». Sans en offrir de justification racialiste, Schuchardt identifie un nombre de traits grammaticaux du parler de ce groupe tout en reconnaissant qu'une partie de ces traits se trouve aussi dans les variétés d'autres couches sociales. Contrairement à Auguste Vinson ou Charles Baissac, le professeur autrichien et le juriste réunionnais ne schématisent pas la société créole en fonction des collectifs raciaux préconceptualisés.

Toutefois, il faut considérer dans le travail de Focard une dimension qu'on pourrait caractériser comme racialisme social intra-français. Déjà quelques décennies avant, l'importante œuvre d'Arthur de Gobineau, l'Essai sur l'inégalité des races humaines (1854), véhiculait l'idée que la société française serait composée d'éléments inégaux. À l'instar d'une grande narration racialiste des cultures du monde, vouées à la décadence entraînée par l'impureté des races, la population française comporterait, elle aussi, une partie élevée et forte - la noblesse à prétendue origine romaine - et une partie faible et inférieure - la couche populaire et paysanne à origine gauloise. Dans la pensée de Gobineau, la conception de ces deux classes de la société était éminemment racialiste, étant donné qu'elle se fondait sur une généalogie biologique qui aurait engendré et déterminé les structures sociales (Messling, sous presse). D'une manière similaire, Focard aurait pu penser à une séparation entre Petits Blancs et propriétaires privilégiés, associée aux généalogies celte et romaine respectivement. Ceci aurait ouvert à Focard la possibilité d'expliquer, sur la base d'arguments racialistes, pourquoi les Petits Blancs parlent créole tandis que le reste de la population d'origine européenne a su garder le français. Si un tel fondement de la pensée focardienne reste possible théoriquement, il paraît toutefois improbable non seulement à cause de sa philosophie globalement universaliste qui exclut des réflexions déterministes, mais aussi à cause de l'image d'une société égalitaire que peint Focard. 
Focard met en exergue une image égalitaire de la société qui va jusqu'à la fin du bouleversement politique du XIX ${ }^{\mathrm{e}}$ siècle, inauguré par l'abolition de l'esclavage et achevé - théoriquement - par l'égalité absolue des droits, nonobstant les différences ethniques. Ceci se traduit avant tout dans son style :

Je suis convaincu, du reste, que ces mots ma, me, mi, mou, moua, ti, ta, toua, sont le résultat d'une corruption, d'une altération du langage Bourbon, dont la responsabilité incombe évidemment à MM. nos concitoyens, natifs de la côte d'Afrique. (Focard 1885, p. 218)

Cette responsabilité se situe clairement du côté des "Noirs », plus spécifiquement des esclaves venant du continent africain et non pas de Madagascar, mais il ne faut pas confondre le terme « responsabilité » avec « culpabilité». Focard n'explique qu'un seul trait grammatical et il essaie d'en tracer les sources en les attribuant à la seconde phase de sa théorie de la créolisation : celle du passage du langage pré-créolisé des Européens aux esclaves.

Si Focard parle toujours de " corruption », il apparait qu'il se corrige tout de suite en disant « altération », expression qui lui semble plus appropriée - il faut se rappeler que l'article est la version écrite d'un discours devant la société savante. Ensuite, Focard parle de «MM. nos concitoyens », tournure qu'il répète à plusieurs reprises partout dans le texte et qui n'est pas du tout ironique. Aux yeux de Focard, les locuteurs du créole, qu'ils soient noirs ou blancs, ne méritent donc pas le dédain que leur attribuent souvent les contemporains. De surcroît, Focard ne parle pas des « Noirs» tout court, mais il examine toujours un groupe bien défini, dans le cas présent les descendants des esclaves mozambicains qui étaient minoritaires par rapport à ceux d'origine malgache et qui avaient ainsi une position sociale à part. Pour les autres créolistes, de telles subtilités sont vaines car tous les esclaves et leurs héritiers rentrent dans la catégorie des " Noirs", peu importe leur histoire linguistique, culturelle, ou leur origine géographique. $\mathrm{Au}$ lieu des conditions raciales, Focard postule donc l'éducation, ou plutôt le manque d'éducation, comme facteur décisif de la créolisation. Selon le modèle courant en créolistique, un meilleur système éducatif n'aurait en rien pu empêcher l'émergence du créole car les « Noirs » n'auraient pas eu les capacités nécessaires pour pouvoir profiter de la scolarisation. Dans la perspective du déterminisme racialiste prédominant, toute tentative d'éducation ou de développement aurait échoué. L'idée même de «mission civilisatrice » connaît ainsi une crise profonde. Cette crise ne touche pas Focard. Pour lui, tout dépend d'une régulation et d'un encadrement linguistiques : si on avait «montré à lire », comme l'écrit Focard, la langue créole ne se serait pas formée.

En situant le processus de créolisation dans le domaine des enfants, l'approche 
de Focard fait penser aux positions universalistes actuelles qui, elles aussi, mettent l'accent sur les modes d'acquisition de la langue pendant l'enfance. Ceci est d'autant plus apparent que Focard parle d'un instinct dont disposeraient les locuteurs du créole : « Et pourtant, lorsqu'on entend le langage si précis, si clair de nos indigènes, on dirait qu'ils ont l'instinct du "pouvoir d'un mot mis en sa place". » (1885, p. 193). Toutefois, l'universalisme de Focard prend une forme différente. D'abord il faut noter qu'il ne parle pas des enfants à l'âge préscolaire mais bien des élèves. Ceux-ci auront donc déjà acquis une forme de langage et il ne leur faudra pas activer une grammaire universelle ou un " programme biologique » pour compléter une grammaire mal transmise, comme le soutiennent les partisans de cette théorie de la créolisation depuis le travail-clé de Bickerton $(1981)^{16}$. Selon Focard, le langage des enfants n'attend que son façonnement par le système éducatif. Il s'agit ici non pas d'un universalisme cognitif mais plutôt d'un universalisme bien classique qui part de l'image de la formation de l'homme. Celuici dispose d'une faculté de langage, caractérisée comme « instinct » par Focard et dont disposent les « Noirs » autant que les «Blancs », et l'homme peut être élevé par le savoir, par un processus d'éducation qui ne façonne pas seulement son esprit mais, le cas échéant, aussi son langage. Ce serait sans doute aller trop loin que de pousser une telle comparaison avec les théories actuelles dans la mesure où les deux approches universalistes ne se rencontrent qu'à un niveau très général quoiqu'essentiel : celui de la conviction d'une aptitude égale de l'humanité au développement linguistique. Il faut faire une distinction claire et nette entre l'universalisme classique de Focard et l'universalisme du Portugais Adolpho Coelho, contemporain de Focard, qui anticipe beaucoup plus clairement les idées du cognitivisme universaliste de la créolistique actuelle (Sousa 2014 ; Krämer 2014b).

C'est dans le cadre de ces idées universalistes qu'on peut interpréter le terme « instinct » chez Focard, bien qu'il puisse prendre des connotations très diverses dans la créolistique du XIX ${ }^{\mathrm{e}}$ siècle. Auguste Vinson, lui, parle des créolophones - et donc des « Noirs » tout court - en tant que « naturels » $(1883$, p. 25) qui suivraient un « instinct sauvage » $(1883$, p. 17 et 19) qui les aurait menés à des altérations de la langue française, altérations d'ailleurs inévitables car dépendantes des conditions raciales. De même, Louis Ducrocq (1902, p. 450), dans sa brève description du créole mauricien, parle du « noir livré aux instincts grossiers de sa race ». Comme le montrent ces deux cas, l'idée de l'instinct était donc associée couramment à la conception de l'homme « Noir » comme primitif et guidé par ses sens et besoins naturels par opposition au «Blanc » civilisé, réfléchi et raisonnable. Inconsciemment, Focard embraye sur une réappropriation du terme dans le sens universaliste après qu'il avait été transformé en élément constitutif de l'idéologie du déterminisme racial.

$16 C f$. aussi la critique développée dans Krämer (2014a, p. 203-206), Ennis et Pfänder (2013, p. 191-216) et Véronique (2007). 


\section{KOMAN FOCARD I ÉKRI KRÉOL, ÉPI KOMAN I DÉKRI ALI ? ${ }^{17}$}

Au niveau de la description grammaticale, le travail de Focard reste peu systématique. De manière assez éclectique, il mélange des phénomènes phonologiques ou morphologiques avec des réflexions sur les étymologies de certains lexèmes créoles perdus ou anachroniques dans le français contemporain et censés souligner le caractère « acrolectal» de certaines expressions. Parfois, Focard donne des interprétations sémantiques en créant un cadre presque narratif qui décrit, pour chacun des mots, son usage, son contexte phraséologique et ses connotations ${ }^{18}$. Pourtant, il observe déjà bon nombre de caractéristiques confirmées par la recherche actuelle. C'est ainsi qu'il discute des simplifications des groupes consonantiques comme capabe $<$ capable $>$ dans le même paragraphe que les procopes (ranzé < arranger) et les formes clitiques des pronoms personnels avec des marqueurs préverbaux comme « $m a$ pour moin, ta pour toué ». (1885, p. 192). Focard mentionne bien quelques traits morphologiques, dont par exemple le manque de distinctions ouvertes entre singulier et pluriel. Par ailleurs, il relève deux procédés de négation : "Pour indiquer qu'une chose n'existe pas, ils diront n'a point; et pour une chose seulement absente, ils diront n'a pas.» (1885, p. 227). Il reste difficile de confirmer si cela n'est qu'une spéculation de Focard ou bien s'il se fonde sur sa compétence linguistique de Réunionnais. Il est bien probable que les négations par pas et point aient été, à l'époque comme aujourd'hui, où point est de moins en moins fréquent, des variantes sans distinction de ce genre. Focard donne lui-même un exemple dans lequel il n'observe pas cette règle : « [...] vous l'ablye à moin ? - N'a point, moin n'a pas blye à vous $[. ..] »^{19}$. Focard cherche sans doute à expliquer cette variation par une régularité. Cette volonté est pourtant significative car d'autres créolistes de l'époque auraient probablement mis ce phénomène au compte de l'image de la prétendue non-grammaticalité du créole et de l'incapacité des locuteurs à observer des structures linguistiques régulières.

Sinon, Focard s'intéresse principalement aux particularités phonétiques et c'est au niveau de la structure phonologique que son texte se prête à des analyses plus détaillées ${ }^{20}$. Comme il attribue certaines "préférences " phonétiques à des groupes ethniques particuliers, il serait intéressant d'établir s'il ne s'agit là que d'impressions subjectives ou bien s'il y a des fondements historiques qui s'expliqueraient par les apports phonologiques des différentes langues des esclaves ou par d'autres niveaux de variation phonologique. Il faut noter, cependant, que Focard attribue bon nombre de processus morphosyntaxiques à une prétendue

17 «Comment Focard écrit-il le créole, et comment le décrit-il ?»

18 Voir, à titre d'exemple, Focard (1885, p. 185-189).

19 Focard (1885, p. 215). Passage cité sans italiques. « Vous m'avez oublié ? - Non, je ne vous ai pas oublié. »

20 À propos de l'attention prédominante portée à la phonologie et à la graphématique dans la créolistique réunionnaise de l'époque $c f$. Vintilă-Rădulescu (1976, p. 126-128). 
variation phonétique sans en expliquer en profondeur les raisons grammaticales. C'est le cas, par exemple, pour son analyse des différences entre $m a$ et $m i$ qui sont le résultat d'une clitisation du pronom avec la particule préverbale - phénomène que Focard ne développe pas en détail ${ }^{21}$ et qu'il attribue notamment à une influence des locuteurs du continent africain. Ceci dit, Focard identifie tout à fait les marqueurs non-clitiques comme té, $i$ ou $l a^{22}$.

L'intérêt primordial de Focard pour la phonétique est peu surprenant, étant donné que sa réflexion linguistique est fortement ancrée dans la primauté de la langue écrite et qu'il voit dans la phonologie créole avant tout une différence de prononciation, donc tout à fait un effet phonétique, par rapport à ce que prescrit l'orthographe française. Pour n'en citer qu'un seul exemple : " Ils [les noirs indigène ${ }^{23}$ ] ont une prédilection telle pour le $z$ ' qu'ils lui font remplacer plusieurs lettres de notre alphabet » $(1885$, p. 190).

En effet, Focard a une idée tout à fait normative de la langue. Il se livre à une critique détaillée des fables créoles de Louis Héry (1883) et de certains de ses contemporains qui écrivent en créole. Tout en admettant que certains passages ne sont pas « facile[s] à créoliser » (Focard 1885, p. 206), Focard examine en détail l'orthographe des autres auteurs pour souligner «l'impérieuse nécessité d'écrire les mots de notre patois, selon leur prononciation propre » $(1885$, p. 216). C'est dans ces débats sur le langage écrit qu'il faut constater des similarités avec le travail d'Auguste Vinson, en dépit du désaccord profond relatif à sa conception de la différence humaine. Vinson, comme la plupart des créolistes contemporains, se pose la question de l'orthographe créole :

[...] j'éprouve une sérieuse difficulté à l'écrire. Pourquoi ? Parce que l'orthographe, objet de conventions dans toutes les langues, n'est pas, à mon avis, bien établie pour ce patois. Le genre n'y existe pas, [...], le verbe n'a pas de nombre, il est toujours au singulier [...], enfin mille imperfections semblables, qui doivent nécessairement exister pour une langue, qui n'a pas encore de grammaire qui en règle les conditions, si vous aimez mieux, les conventions. (A. Vinson 1883, p. 22)

Pour Vinson, la norme grammaticale et les règles d'orthographe paraissent précéder les régularités de la langue. Il n'y a pas de structure proprement dite

$21 C f$. Focard (1885, p. 222-223) où il est question du « pronom personnel vous, qui se change parfois en vou y » sans aucune explication des règles morphosyntaxiques qui sont à la base de cette construction.

22 Focard (1885, p. 218). Cf. aussi Chaudenson (1981, p. 187).

23 Focard parle fréquemment des " noirs indigènes ", expression assez remarquable vu que l'île de la Réunion était inhabitée avant l'ère coloniale et que les esclaves n'étaient donc pas plus « indigènes » que les Européens. Cette expression reprend ainsi une logique établie par l'expérience coloniale dans le continent africain où " indigène » est devenu quasiment synonyme de " noir » au point de former ce terme fixe employé par Focard. Vu que le terme noir a perdu en partie sa connotation ethnico-raciale à la Réunion et que celle-ci a été remplacée par une dimension sociale, l'expression « noirs indigènes » n'est pourtant pas, dans ce contexte, purement tautologique. (Chaudenson 1974, p. 88) 
tant que les savants ne la créent pas. En même temps, Vinson ne distingue pas clairement entre langue écrite et langue parlée. La création des normes écrites est censée entraîner sinon établir des règles de la langue parlée, et ces règles englobent des régularités grammaticales au même titre que des normes du bon usage. Selon la conception courante et établie longtemps avant Vinson et Focard, afin de devenir une langue proprement dite, tout idiome parlé doit principalement devenir une langue écrite ${ }^{24}$. En faisant cet amalgame entre normes établies et régularités naturelles, Vinson soumet la grammaire à l'orthographe. Idem pour Focard quand il déplore que « les petits créoles abusent de l'accent grave ». Ceci serait possible uniquement à condition que les locuteurs conçoivent toujours une image du mot écrit avant de le prononcer. Une telle idée de la langue comme ensemble d'unités normées écrites va de pair avec une perspective essentiellement lexicocentriste. À propos de la question de la mixité du créole, Forcard écrit :

Que le créole soit un français altéré, je le veux bien ; mais qu'il ait été créé avec des mots mêlés de cafre, de malgache et d'indien, je puis d'autant moins l'admettre que je ne trouve aucun de ces mots là dans sa phraséologie générale. [...] à induire de là que notre patois créole descend des insulaires de la côte d'Afrique et de Madagascar, il y a loin. (Focard 1885, p. 181)

Pour répondre à cette question - et pour réagir donc à l'une des problématiques principales du XIX ${ }^{e}$ siècle : celle de la pureté perdue des civilisations européennes Focard ne considère que le niveau lexical, bien que son texte contienne une grande partie décrivant bien d'autres parties de la grammaire. Quelques années plus tard, Adolphe Dietrich va écrire son étude comparée des créoles de La Réunion et de l'île Maurice en tenant compte entre autres du texte de Focard dont il dit : «L'étymologie est son point de mire. » (Dietrich 1891, p. 222). Plusieurs décennies après Schlegel, Focard n'assume pas la primauté de la structure interne (" innre Structur »; Schlegel 1808, p. 28). Ainsi, le caractère de la langue est toujours défini par une forme de généalogie et on peut compter Focard parmi les premiers créolistes à représenter une position superstratiste. Cette généalogie est cependant exclusivement lexicale et donc intralinguistique sans qu'elle soit liée directement à une généalogie biologique, voire raciale. Par conséquent, l'idée que le créole serait « tout français », et donc pas une langue mixte, n'implique pas que la mixité d'une langue déclencherait en même temps sa décadence irrémédiable. Même dans la description grammaticale, Focard admet très rarement qu'un phénomène ne trouve pas son explication dans l'influence du français. Dans de tels cas, il évoque vaguement des « préférences » des créateurs du créole sans spécifier où il faudra chercher l'origine de ces préférences. On ne saurait que spéculer sur le rapport de telles préférences avec la notion essentialiste du " génie » évoquée beaucoup plus souvent dans les travaux philologiques de l'époque et qui se

$24 C f$. le concept du « devenir langue » (Sprachwerdung) du créole introduit par Bachmann (2005). 
manifesterait dans les langues des différentes cultures ou races du monde : est-ce que les préférences sont des choix conscients tandis que le génie est une entité métaphysique qui échappe à la connaissance explicite des locuteurs ? La préférence est-elle une disposition sociale acquise par opposition au génie qui, dans le contexte de la philologie racialiste du XIX ${ }^{e}$ siècle du moins, est une disposition biologique et donc héréditaire (Trabant 2012, p. 251-257 ; Meschonnic 1997, p. 178) ? Si l'on peut faire ressortir beaucoup de contrastes entre le travail de Focard et le discours prédominant, c'est dans de telles questions terminologiques et celles des conceptions associées que le texte commence à devenir plutôt brumeux.

\section{KOSA FOCARD I APRANN ANOU $?^{25}$}

Volcy Focard introduit des arguments qui seront plus tard des éléments importants du principe fondateur qui explique la créolisation à partir des structures sociolinguistiques dominées par des locuteurs de variétés non-standard de la langue européenne (Mufwene 1996). C'est dans ce contexte que « les enfants créoles ont eu un rôle catalyseur dans la cristallisation des structures de leurs nouveaux vernaculaires » (Mufwene 2005, p. 150), structures qui émergent d'une sorte de « re-création » (p. 191-192) dans la transmission des formes pré-créolisées. Si le modèle de Focard avec ses deux phases reste trop simplifié et que le manque d'une scolarisation formelle ne saurait être la cause unique de la créolisation, cette réflexion préfigure déjà certaines idées actuellement exprimées dans la branche de la créolistique qui se fonde sur les approches de l'écologie du langage.

Robert Chaudenson reprend et nuance les classifications focardiennes des différentes variétés créoles selon les groupes ethnico-linguistiques de l'île pour en tirer des conclusions sur la variation structurelle interne du créole (Chaudenson 2000, p. 364-365 ; 1981, p. 69-70, 162-167). Si les langues substratiques ne sont pas nécessairement à la base de cette différenciation, nous pouvons néanmoins relever, par le biais du travail de Focard, un éventail historique de variétés qui s'est perdu ou réorganisé depuis la décolonisation.

Focard n'apporte pas nécessairement des informations décisives sur le développement grammatical du créole réunionnais, pourtant, il occupe une place importante dans l'histoire de la recherche sur les créoles dans la mesure où il prend une position remarquable au sein du champ scientifique contemporain : c'est lui qui, comme très peu d'autres créolistes de l'époque, arrive à décrire une langue créole sans avoir recours au modèle discursif prédominant ancré dans le racialisme de l'époque. Focard a été lu et cité par d'autres créolistes comme Armand Corre qui met à profit sa vue d'ensemble des langues créoles dans son pamphlet contre les 
habitants des colonies, sans tenir compte des nuances et de la prudence qu'apporte Focard lui-même (Corre 1890).

Ce n'est pas exclusivement en tant que grammairien que la mémoire créoliste devrait garder le souvenir de Focard, mais plutôt en tant que premier « sociolinguiste » qui a introduit, avec Hugo Schuchardt et son élève Adolphe Dietrich, la dimension sociale de la créolisation ainsi que la variation socio-ethnique sans aucune dérive racialiste.

Focard est un personnage exceptionnel à deux égards : d'abord il tient à une idée de la langue qui peut paraître un peu dépassée à une époque où la linguistique moderne commence à se former et où d'autres créolistes savent bien faire la différence entre le son et sa représentation écrite. Si Focard met l'accent sur le lexique, il renvoie à la pratique philologique des siècles précédents. Ensuite, il est extraordinaire et remarquable que Focard arrive à échapper grosso modo au discours racialiste de l'époque. Ces deux dimensions vont sans doute de pair : Focard suit un modèle classique et donc une idée de la langue bien ancrée dans la philosophie pré-dix-neuvièmiste. Celle-ci n'a pas nécessairement été antiraciste, mais en sauvegardant le principe de l'universalisme, le risque de classer l'homme dans une hiérarchie absolue et surtout perpétuelle selon un modèle déterministe semble limité.

En même temps, le travail de Focard surprend par ses positions progressistes, voire avant-gardistes qui mettent au cœur de la réflexion non les collectifs raciaux mais les structures complexes d'une société qui ne peut pas être décrite avec une simple logique binaire. Même s'il n'évite pas complètement les stéréotypes courants, c'est surtout par son accent sociolinguistique avant la lettre que Focard se démarque de la plupart des autres créolistes de l'époque.

Ainsi, ce seul texte peu étudié fait ressortir que la créolistique dispose en quelque sorte d'un héritage du désaccord : dès le début de son émergence comme discipline scientifique, les travaux suivent des orientations discursives très diverses. Cette diversité se manifeste notamment dans la comparaison des deux travaux de Vinson et de Focard, textes qui ont été écrits pratiquement au même moment, au même endroit, par deux auteurs qui se connaissaient, et qui sont toutefois arrivés à des modèles virtuellement opposés.

S'il y a aujourd'hui dans la communauté des créolistes un certain nombre de questions controversées, parfois avec des dimensions idéologiques, on peut se rappeler que le désaccord a été productif dans le passé. Il a protégé certains auteurs comme Focard des forces écrasantes du discours racialiste qui était presque incontournable à l'époque.

Les créolistes «non-racialistes » comme Focard ou Schuchardt sont-ils vraiment des irréductibles au milieu d'une philologie racialiste ? Ils sont certes minoritaires, et ils n'arrivent pas, à l'époque, à fragiliser le modèle de réflexion prédominant. Pourtant, ils nous enseignent deux idées essentielles : autrefois comme de nos jours, esquiver les arguments du discours prédominant est une 
possibilité réelle. Si la thèse saïdienne reste valide aussi en créolistique, si donc la philologie du XIX ${ }^{\mathrm{e}}$ siècle faisait cause commune avec le régime colonial et ses structures de domination (Said 1978), cette alliance n'a jamais été sans alternative. Pareillement, dans la pratique scientifique de nos jours, nous sommes invités à garder un haut niveau de réflexivité en mettant en cause les aprioris de nos approches - et en mettant en cause les aprioris du discours extrascientifique. C'est surtout dans l'opinion publique sur les créoles et toutes les langues réputées déficientes ou inférieures que des structures argumentatives du XIX ${ }^{\mathrm{e}}$ siècle persistent. Dans une perspective historique, l'exemple de Focard peut contribuer à défier de telles conceptions hiérarchisantes.

En outre, une étude historique peut nous donner des indices fructueux pour les débats actuels dans la linguistique créole, notamment la question de savoir si les créoles constituent ou non une classe exceptionnelle de langues. Ceux qui partagent cette hypothèse se fondent essentiellement sur des explications grammaticales (McWhorter 2011, p. 124). Focard nous enseigne, cependant, que la dimension sociale et socio-historique ne doit pas être oubliée pour arriver à une image complète et appropriée des langues créoles. S'il est, selon Versteegh (2004, p. 305), " generally held that the real beginnings of this field are marked by two conferences organized in Jamaica » en 1959 et 1961, il faut reconnaitre qu'un grand nombre de questions principales de recherche sont déjà présentes bien avant, dont non seulement les considérations proto-sociolinguistiques de Focard mais aussi le substratisme de Lucien Adam ou le superstratisme de René de PoyenBellisle (Krämer 2013a). Relire ces sources du temps de la genèse de la créolistique comme discipline académique - dont le sérieux ne saurait être nié et dont l'ancrage dans les institutions universitaires ne devrait être sous-estimé - pourra ainsi nous aider à rééquilibrer les discussions dans cette même discipline à l'heure actuelle (Krämer 2013b, p. 107-108). Cela nous permettra de mieux apprécier à quel point des théories de la créolisation jugées novatrices reposent souvent sur des aprioris et des idées exprimées il y a 130 ans environ.

\section{BIBLIOGRAPHIE}

Bachmann, Iris, 2005. Die Sprachwerdung des Kreolischen. Eine diskursanalytische Untersuchung am Beispiel des Papiamentu, Tübingen, Narr.

Baissac, Charles, 1880. Étude sur le patois créole mauricien, Nancy, Berger-Levrault et Cie. Bickerton, Derek, 1981. Roots of Language, Ann Arbor, Karoma.

Bourquin, Alexandre, 2005. Histoire des Petits-Blancs de La Réunion, Paris, Karthala.

Chaudenson, Robert, 1974. « Le Noir et le Blanc : la classification raciale dans les parlers créoles de l'océan Indien », Revue de linguistique romane, 38, 75-94.

- 1981. Textes créoles anciens. (La Réunion et île Maurice) Comparaison et essai d'analyse, Hambourg, Buske. 
- 2000. «Créolisation du français et francisation du créole : le cas de Saint-Barthélemy et de la Réunion », in Ingrid Neumann-Holzschuh \& Edgar Schneider (éd.), Degrees of Restructuring in Creole Languages, Amsterdam, Benjamins, 361-381.

Corre, Armand, 1890. Nos Créoles, Paris, Albert Savine.

Dietrich, Adolphe, 1891. «Les parlers créoles des Mascareignes », Romania, 20, 216-277.

Ducrocq, Louis, 1902. "Idiome enfantin d'une race enfantine. Le créole de l'Ile de France », Revue de Lille, XIIIe année, tome V (1901-1902), mai 1902, 439-458.

Ennis, Juan Antonio et Pfänder, Stefan, 2013. Lo criollo en cuestión. Filología e historia, Buenos Aires, Katatay.

Focard, Volcy, 1862. "Troubles de Saint-Louis en 1848 », Bulletin de la Société des sciences et arts de l'île de la Réunion, année 1862, 7-18.

- 1885. « Du patois créole de l'île Bourbon », Bulletin de la Société des sciences et arts de l'île de la Réunion, année 1884, 179-239.

Gobineau, Arthur, 1854. Essai sur l'inégalité des races humaines [Réédition Paris, FirminDidot, 1940].

Héry, Louis, 1883. Esquisses africaines. Fables créoles et explorations dans l'intérieur de l'île Bourbon, Paris, J. Rigal et Cie.

Krämer, Philipp, 2012. " Hugo Schuchardt im Zentrum der frankophonen Kreolistik. Korrespondenzen mit Lucien Adam, Volcy Focard, Alfred Mercier, Alcée Fortier und René de Poyen-Bellisle », Grazer Linguistische Studien, 78, 129-156.

- 2013a. « Vom Instinkt zum Bioprogramm, von der Mischung zum Hybrid. Historische und gegenwärtige Vorstellungen von Kreolisierung als Wandelprozess in der Sprache », Gesine Müller \& Natascha Ueckmann (éd.), Kreolisierung revisited. Debatten um ein weltweites Kulturmodell, Bielefeld, transcript, 43-63.

- 2013b. « Creole exceptionalism in a historical perspective - from 19th century reflection to a self-conscious discipline », Language Sciences, 38, 99-109.

- 2014a. Die französische Kreolistik im 19. Jahrhundert. Rassismus und Determinismus in der kolonialen Philologie, Hambourg, Buske.

- 2014b. « Neither raça nor povo: Adolpho Coelho's particular universalism », idem (éd.), Ausgewählte Beiträge der Kreolistik des 19. Jahrhunderts. Emilio Teza, Thomas Russell, Erik Pontoppidan, Adolpho Coelho, Hambourg, Buske, 175-184.

McWhorter, John H. , 2011. Linguistic Simplicity and Complexity: Why do Languages Undress?, Boston/Berlin, DeGruyter.

Meschonnic, Henri, 1997. La langue française. Essai sur une clarté obscure, Paris, Hachette [Réédition 2001].

Messling, Markus, 2012. «Philologie et racisme. À propos de l'historicité dans les sciences des langues et des textes », Annales. Histoire, Sciences sociales, 67/1, 153-182.

- sous presse. "Anthropologie, Kultur und Sprache: Gobineaus rassenlogischer Materialismus ", in Philipp Krämer, Markus Lenz und Markus Messling (éd.), Rassedenken in der Sprach- und Textreflexion. Kommentierte Grundlagentexte des 19. Jahrhunderts, Munich, Fink.

Mufwene, Salikoko, 1996. " The Founder Principle in Creole Genesis », Diachronica, 13/1, 83-134.

- 2005. Créoles, écologie sociale, évolution linguistique, Paris, L'Harmattan.

Poyen-Bellisle, René de, 1894. Les sons et les formes du créole dans les Antilles, Baltimore, John Murphy \& Co.

Said, Edward W., 1978. Orientalism. Western Conceptions of the Orient [Réédition Londres, Penguin, 2003].

Schlegel, Frierich, 1808. Ueber die Sprache und Weisheit der Indier. Ein Beitrag zur Begründung der Alterthumskunde, Heidelberg, Mohr und Zimmer.

Schuchardt, Hugo, 1882. «Sur le créole de la Réunion », Romania, 11, 589-593.

Sousa, Silvio Moreira de, 2014. "Die letzten Geheimnisse Adolfo Coelhos? ", in Philipp Krämer (éd.), Ausgewählte Beiträge der Kreolistik des 19. Jahrhunderts. Emilio Teza, Thomas Russell, Erik Pontoppidan, Adolpho Coelho, Hambourg, Buske, 157-174. 
Stein, Peter, 2005. «Hugo Schuchardt und die portugiesischen Kreolsprachen », Endruschat, in Annette Endruschat und Axel Schönberger (éd.), Portugiesische Kreolsprachen: Entstehung, Entwicklung, Ausbau und Verwendung, Frankfurt (Main), Domus Editoria Europea, 201-215.

Todorov, Tzvetan, 1989. Nous et les autres. La réflexion française sur la diversité humaine, Paris, Le Seuil.

Trabant, Jürgen, 2012. Weltansichten. Wilhelm vom Humboldts Sprachprojekt, Munich, Beck.

Véronique, Daniel, 2007. «Des racines du langage : La linguistique naturaliste de Derek Bickerton », Histoire Épistémologie Langage, XXIX, 2, 163-176.

Verrier, Anatole-Joseph, 1906. « Le patois créole de l'île de la Réunion », Mémoires de la Société nationale d'Agriculture, Science et Arts d'Angers, tome IX, année 1906, 283305.

Versteegh, Kees, 2004. "The future of creolistics », in Piet van Sterkenburg (éd.), Linguistics Today - Facing a Greater Challenge, Amsterdam, Benjamins, 305-319.

Vinson, Auguste, 1883. "Les origines du patois de l'île Bourbon », Bulletin de la Société des sciences et arts de l'île de la Réunion, année 1882, 88-129 [Langue créole de La Réunion, Textes Anciens, vol. 1, présenté et annoté par Monique Payet, Saint-Denis de La Réunion, Association Tangol, 2006].

- 1885. «Étude sur Hæckel. Le Lamarckisme », Bulletin de la Société des sciences et arts de l'île de la Réunion, année 1884, 87-96.

Vinson, Julien, 1884. "Créoles (linguistique) », in Louis-Adolphe Bertillon et al. (éd.), Dictionnaire des sciences anthropologiques, Paris, Doin, 345-347.

Vintilă-Rădulescu, Ioana, 1976. Le créole français, La Haye, Mouton.

Wolf, Michaela, 1993. Hugo Schuchardt Nachlass. Schlüssel zum Nachlass des Linguisten und Romanisten Hugo Schuchardt (1842-1927), Graz, Leykam. 